\title{
Spin Quintet in a Silicon Double Quantum Dot: Spin Blockade and Relaxation
}

\author{
Theodor Lundberg $\odot,{ }^{1,2, *}$ Jing Li $\odot,{ }^{3}$ Louis Hutin, ${ }^{4}$ Benoit Bertrand, ${ }^{4}$ David J. Ibberson $\odot,{ }^{5,6}$ Chang-Min Lee, ${ }^{7}$ David \\ J. Niegemann, ${ }^{8}$ Matias Urdampilleta $\odot,{ }^{8}$ Nadia Stelmashenko, ${ }^{7}$ Tristan Meunier, ${ }^{8}$ Jason W. A. Robinson, ${ }^{7}$ Lisa Ibberson, ${ }^{2}$ \\ Maud Vinet, ${ }^{4}$ Yann-Michel Niquet, ${ }^{3}$ and M. Fernando Gonzalez-Zalba ${ }^{2, \dagger, \$}$ \\ ${ }^{1}$ Cavendish Laboratory, University of Cambridge, J.J. Thomson Avenue, \\ Cambridge CB3 OHE, United Kingdom \\ ${ }^{2}$ Hitachi Cambridge Laboratory, J.J. Thomson Avenue, Cambridge CB3 OHE, United Kingdom \\ ${ }^{3}$ Université Grenoble Alpes, CEA, IRIG, MEM/L_Sim, 38000 Grenoble, France \\ ${ }^{4}$ CEA/LETI-MINATEC, CEA-Grenoble, 38000 Grenoble, France \\ ${ }^{5}$ Quantum Engineering Technology Labs, University of Bristol, \\ Tyndall Avenue, Bristol BS8 1FD, United Kingdom \\ ${ }^{6}$ Hitachi Cambridge Laboratory, J.J. Thomson Avenue, Cambridge CB3 OHE, United Kingdom \\ ${ }^{7}$ Department of Materials Science and Metallurgy, University of Cambridge, \\ 27 Charles Babbage Road, Cambridge CB3 OFS, United Kingdom \\ ${ }^{8}$ CNRS, Grenoble INP, Institut Néel, Université Grenoble Alpes, 38000 Grenoble, France
}

(Received 3 December 2019; revised 11 April 2020; accepted 5 August 2020; published 14 October 2020)

\begin{abstract}
Spins in gate-defined silicon quantum dots are promising candidates for implementing large-scale quantum computing. To read the spin state of these qubits, the mechanism that has provided the highest fidelity is spin-to-charge conversion via singlet-triplet spin blockade, which can be detected in situ using gate-based dispersive sensing. In systems with a complex energy spectrum, like silicon quantum dots, accurately identifying when singlet-triplet blockade occurs is hence of major importance for scalable qubit readout. In this work, we present a description of spin-blockade physics in a tunnel-coupled silicon double quantum dot defined in the corners of a split-gate transistor. Using gate-based magnetospectroscopy, we report successive steps of spin blockade and spin-blockade lifting involving spin states with total spin angular momentum up to $S=3$. More particularly, we report the formation of a hybridized spin-quintet state and show triplet-quintet and quintet-septet spin blockade, enabling studies of the quintet relaxation dynamics from which we find $T_{1} \sim 4 \mu \mathrm{s}$. Finally, we develop a quantum capacitance model that can be applied generally to reconstruct the energy spectrum of a double quantum dot, including the spin-dependent tunnel couplings and the energy splitting between different spin manifolds. Our results allow for the possibility of using Si complementary metal-oxide-semiconductor quantum dots as a tunable platform for studying high-spin systems.
\end{abstract}

DOI: $10.1103 /$ PhysRevX.10.041010

\section{INTRODUCTION}

High-spin states have been shown to play a key role in a variety of important physical phenomena. They are involved in singlet fission in organic photovoltaics $[1,2]$, unconventional high-spin superconductivity $[3,4]$, and the

*tw128@cam.ac.uk

†mg507@cam.ac.uk

"Present address: Quantum Motion Technologies, Nexus, Discovery Way, Leeds, LS2 3AA, United Kingdom.

Published by the American Physical Society under the terms of the Creative Commons Attribution 4.0 International license. Further distribution of this work must maintain attribution to the author(s) and the published article's title, journal citation, and DOI.
Subject Areas: Quantum Physics,

Quantum Information,

Semiconductor Physics energy states of molecules and complexes with large delocalized electron systems that are relevant, for example, to biochemical catalysis [5-7]. Similarly, spin-based quantum computing also necessitates a comprehensive understanding of the interplay of the various spin states that exist in the quantum computing platform of choice [8-11]. Spins bound to quantum dots defined in silicon have garnered significant attention as an attractive quantum computing platform due to their long coherence times [12,13], compatibility with industrial manufacturing techniques [14-16], and, recently, the demonstration of high-fidelity one- and two-qubit operations [17-19]. In spin qubits, quantum state readout is achieved via spin-to-charge conversion, which translates the spin state into a selective movement of charge that can be efficiently detected using charge sensors [20] or resonant circuits [21]. Currently, the spin-to-charge mechanism that has enabled the highest 
fidelity readout, even at high temperatures and moderate magnetic fields $[22,23]$, is Pauli spin blockade of the twoelectron singlet and triplet states [24-26]. Under spin blockade, electron transitions from one quantum dot to another are prohibited in the triplet manifold due to the Pauli exclusion principle, while transitions among singlet states are permitted. However, singlet-triplet spin blockade can be lifted by the presence of low-energy excited states that enable direct triplet tunneling and render spin blockade less effective [27]. Given that silicon possesses an additional valley degree of freedom [28], the energy spectrum in silicon quantum dots can be rather complex, and accurately identifying when singlet-triplet blockade occurs is hence of importance for achieving reliable and scalable readout of spin qubits in silicon.

In this paper, we go beyond the standard description of singlet-triplet Pauli spin blockade and the previous work on multielectron double quantum dots (DQDs) with spin angular momentum $S \leq 1$ [29,30]. Here, we explore multiparticle valence states with large spin angular momentum and demonstrate that low-lying excited states can lead to successive steps of spin blockade and spin-blockade lifting, which span spin states with total spin angular momentum up to $S=3$, thereby expanding concepts introduced with transport measurements in Ref. [31]. We do so using a DQD defined electrostatically in the corners of a $\mathrm{Si}$ complementary metal-oxide-semiconductor (CMOS) splitgate transistor [27]. By using gate-based dispersive readout [32-34] and magnetospectroscopy of an interdot charge transition (ICT) between two quantum dots containing a total of 2 and 14 electrons, respectively, we demonstrate the formation of a hybridized spin-quintet state $(S=2)$ between the quantum dots. We show how spin-quintet tunneling can be blocked at low magnetic fields by the triplet states $(S=1)$ and at high fields by a septet state $(S=3)$. The spin blockade is used to study the quintet spin relaxation to the triplet and septet states, which we find to be of the order of a few microseconds. Moreover, to better understand the magnetic dependence of the dispersive response, we develop a quantum capacitance model that enables reconstruction of the energy spectrum of the coupled DQD, including the spin-dependent tunnel coupling and the energy splitting between different spin manifolds. Overall, our study provides a comprehensive understanding of spin-blockade physics in systems with a dense energy spectrum and allows for the possibility of investigating the dynamics of high-spin systems using programmable CMOS technology.

\section{DISPERSIVE DETECTION OF THE ABSOLUTE DQD ELECTRON OCCUPANCY}

Figure 1(a) shows a SEM micrograph of an $n$-type CMOS split-gate transistor fabricated on a $300-\mathrm{mm}$ siliconon-insulator (SOI) wafer and similar to the device presented here. The inset of Fig. 1(a) presents the cross section of the silicon channel of the transistor, which has a height $h=7 \mathrm{~nm}$, a width $w=70 \mathrm{~nm}$, and is separated from the $\mathrm{Si}$
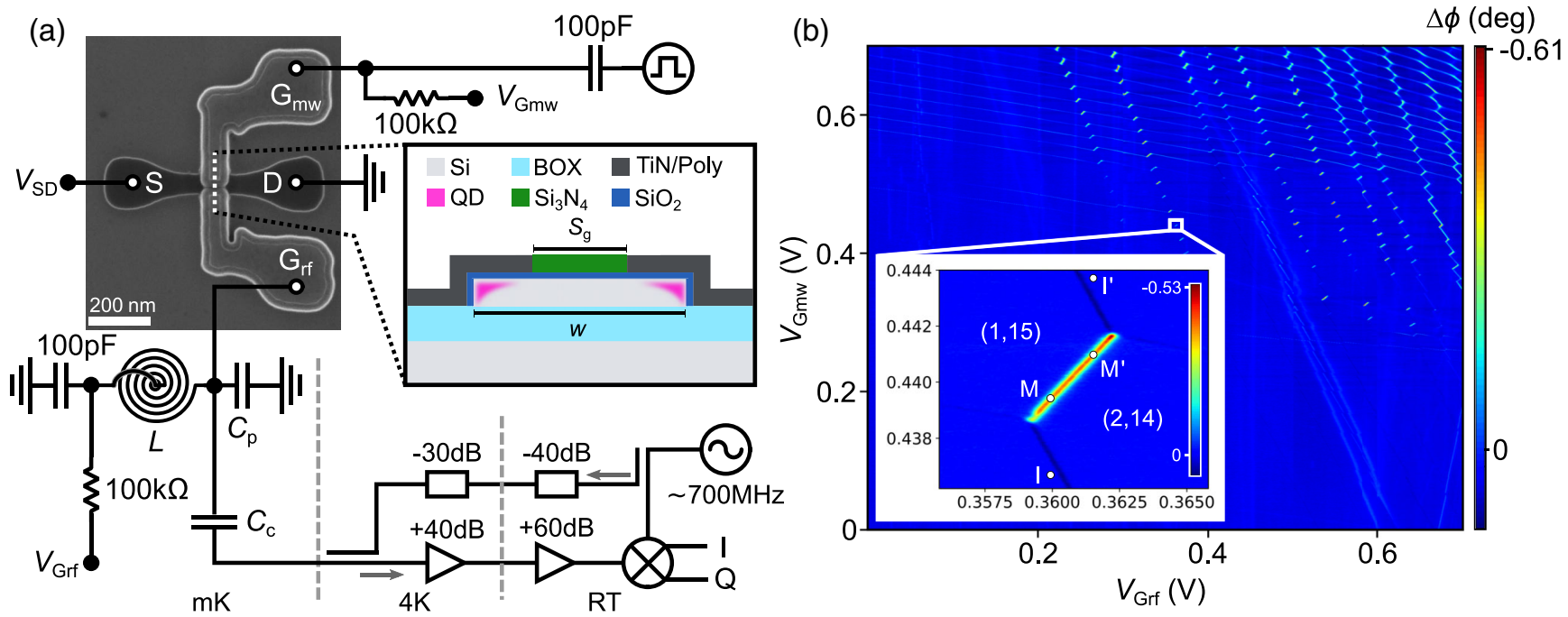

FIG. 1. Measurement setup and charge stability diagram of the DQD. (a) Scanning electron microscopy (SEM) micrograph and sketch of the transistor and measurement setup. The SEM micrograph is taken during processing, after the definition of the $\mathrm{Si}_{3} \mathrm{~N}_{4} \mathrm{spacers} \mathrm{The}$ sketch inset capturing the cross section of the transistor perpendicular to the channel illustrates the architecture of the device as well as the DQD formed in parallel in the channel corners when applying a positive potential to the face-to-face top gates. Gate $\mathrm{G}_{\mathrm{rf}}$ is connected to a resonant LC circuit in which the inductive component is a superconducting NbN planar spiral inductor. (b) Charge stability diagram recorded using gate-based dispersive readout while sweeping the potentials of $\mathrm{G}_{\mathrm{rf}}$ and $\mathrm{G}_{\mathrm{mw}}$, showing regular features characteristic of a DQD. Counting the number of charge transitions in the diagram allows identification of the exact DQD charge configurations, e.g., that shown in the inset, which highlights the $(1,15)-(2,14)$ interdot charge transition investigated in this paper. The points $\mathrm{I}^{\prime} \mathrm{I}^{\prime}, \mathrm{M}$, and $\mathrm{M}^{\prime}$ indicate the initialization and measurement points of pulsing experiments presented in Fig. 3. 
substrate, which we hold at $0 \mathrm{~V}$, by a $145-\mathrm{nm} \mathrm{SiO}_{2}$ buried oxide (BOX). On top and separated from the channel by $6 \mathrm{~nm}$ of $\mathrm{SiO}_{2}$, there is a pair of face-to-face gate electrodes $\left(\mathrm{G}_{\mathrm{rf}}\right.$ and $\left.\mathrm{G}_{\mathrm{mw}}\right)$, which have gate lengths $L_{\mathrm{g}}=60 \mathrm{~nm}$ and are split from each other by $S_{\mathrm{g}}=40 \mathrm{~nm}$. The space between and around the gates is covered by $34-\mathrm{nm}$-wide $\mathrm{Si}_{3} \mathrm{~N}_{4}$ spacers, which help separate the highly $n$-type-doped source (S) and drain (D) contacts from the central part of the intrinsic channel. While it is not of importance to the results described here, we note that the channel region below $\mathrm{G}_{\mathrm{rf}}$ is lightly $\mathrm{Bi}$ doped with an average of about $1 \mathrm{Bi}$ dopant. By increasing the electrostatic potential of $\mathrm{G}_{\mathrm{rf}}$ and $\mathrm{G}_{\mathrm{mw}}$, electrons accumulate in the corners of the channel, thus forming two quantum dots with controllable electron occupancies, $\mathrm{QD}_{\mathrm{rf}}$ and $\mathrm{QD}_{\mathrm{mw}}$, in parallel between the source and drain [see inset of Fig. 1(a)] [35]. Electrons can be drawn in to the quantum dots from the $S$ and $D$ reservoirs, which we hold at $0 \mathrm{~V}$ for all experiments presented in this work.

We control and read the electron configuration of the DQD via the setup presented in Fig. 1(a). The microwave gate $\left(\mathrm{G}_{\mathrm{mw}}\right)$ is connected to a dc line $\left(V_{\mathrm{Gmw}}\right)$ and a highfrequency line through a bias tee. The other gate, the reflectometry gate $\left(\mathrm{G}_{\mathrm{rf}}\right)$, is connected to a dc line $\left(V_{\mathrm{Grf}}\right)$ and, in parallel, to a lumped-element LC resonant circuit comprised of a superconducting $\mathrm{NbN}$ planar spiral inductor as well as the parasitic capacitance to ground, $C_{\mathrm{p}}$. At $B=0 \mathrm{~T}$, the resonance frequency of the resonator is $f_{0}=704.68 \mathrm{MHz}$; however, when increasing the magnetic field, the kinetic inductance of the superconducting spiral inductor increases, leading to shifts in $f_{0}$ according to $f_{0}(B)=1 /\left[2 \pi \sqrt{L(B)\left(C_{\mathrm{p}}+C_{\mathrm{c}}+C_{\mathrm{d}}\right)}\right]$, where $L(B)$ is the magnetic-field-dependent inductance of the spiral inductor, $C_{\mathrm{c}}=0.2 \mathrm{pF}$ is the coupling capacitance, and $C_{\mathrm{d}}$ is the state-dependent device capacitance. We drive the resonator with a frequency $f_{\text {rf }}$ close to resonance and with a power $P_{\mathrm{rf}}$ at the input of the resonator in the range of -105 to $-95 \mathrm{dBm}$. The signal reflected from the resonant circuit is amplified by $40 \mathrm{~dB}$ at $4 \mathrm{~K}$ and by a further $60 \mathrm{~dB}$ at room temperature, where it is subsequently homodyne IQ demodulated and low-pass filtered, thus allowing measurement of the phase shift of the reflected signal, $\Delta \phi$. Variations in phase, $\Delta \phi=-2 Q_{\mathrm{l}} \Delta C_{\mathrm{d}} /\left(C_{\mathrm{p}}+C_{\mathrm{c}}+C_{\mathrm{d}}\right)$, where $Q_{1}$ is the loaded quality factor, arise due to changes in device capacitance that occur, for example, when single electrons tunnel cyclically between QDs, or between a QD and a reservoir, because of the influence of the $\mathrm{rf}$ drive [36].

We present the charge stability diagram in Fig. 1(b) [37] obtained by measuring the phase response as a function of the voltages $V_{\mathrm{Grf}}$ and $V_{\mathrm{Gmw}}$. The hexagonlike features in the diagram confirm the presence of a DQD in the $\mathrm{Si}$ channel, and the lines of nonzero phase shift indicate regions of charge bistability. The short lines with positive slope indicate an electron tunneling between dots, whereas the quasivertical (quasihorizontal) lines correspond to regions where $\mathrm{QD}_{\mathrm{rf}}\left(\mathrm{QD}_{\mathrm{mw}}\right)$ exchanges an electron with the source or drain reservoirs. We note a larger average voltage period of the lines in $V_{\mathrm{Grf}}(\sim 47 \mathrm{mV})$ with respect to $V_{\mathrm{Gmw}}(\sim 18 \mathrm{mV})$, which we attribute to a misalignment of $7 \pm 3 \mathrm{~nm}$ in the placement of the gates on the Si channel in which $G_{m w}$ overlaps the channel more so than $G_{r f}$. The increased elongation and the rounding of the corners of the hexagons at larger gate voltages indicate an increased tunnel coupling between the dots [38]. Gate-based reflectometry readout enables us to discern the addition of the first electron to each of the QDs and to count the addition of subsequent electrons as described in Appendix A. The inset of Fig. 1(b) shows the region of the charge stability diagram around the even-parity interdot charge transition (ICT) between the $(1,15)$ and $(2,14)$ DQD charge configurations. Here, $i$ in $(i, j)$ refers to the electron occupancy of $\mathrm{QD}_{\mathrm{rf}}$, while $j$ refers to the electron occupancy of $\mathrm{QD}_{\mathrm{mw}}$. The tunneling between the two dots results in a change of device capacitance given by the quantum capacitance of the system, which in the slow relaxation limit can be described by the expression [36]

$$
C_{\mathrm{Q}}=-\sum_{i} e \alpha \frac{\partial^{2} E_{i}}{\partial V_{\mathrm{Grf}}^{2}} P_{i},
$$

where $e$ is the electron charge, $\alpha=\alpha_{\mathrm{rf}}-\alpha_{\mathrm{mw}}$ is the difference between the capacitive coupling ratio of $\mathrm{G}_{\mathrm{rf}}$ to $\mathrm{QD}_{\mathrm{rf}}$ and $\mathrm{QD}_{\mathrm{mw}}, E_{i}$ is the eigenenergy of the manyparticle state $i$, and $P_{i}$ is the probability of the state $i$ being occupied. The points I, $\mathrm{I}^{\prime}, \mathrm{M}$, and $\mathrm{M}^{\prime}$ in the inset indicate the initialization and measurement points used for pulsing experiments described later in this paper. In the following, we focus on the region and ICT shown in the inset of Fig. 1(b).

\section{MAGNETOSPECTROSCOPY}

In order to study the spin physics and energy spectrum of the multielectron DQD defined in this device, and, in particular, of the ICT shown in Fig. 1(b), we perform a dispersive magnetospectroscopy study by measuring the line trace intersecting the ICT at $V_{\mathrm{Gmw}}=0.440 \mathrm{~V}$ while increasing the magnetic field $B$, which is applied in plane with the device and at an $83^{\circ}$ angle to the nanowire. To account for the magnetic-field-dependent inductance $L(B)$, which changes significantly for $B \gtrsim 2 \mathrm{~T}$, the magnetospectroscopy study is split into two measurements as presented in Fig. 2(a), where $f_{\text {rf }}$ is adjusted accordingly for $B=2$ to $5 \mathrm{~T}$. The measurements show that the signal seen at $B=0 \mathrm{~T}$ in the inset of Fig. 1(b) disappears when $B>1.2 \mathrm{~T}$ and that a new signal at slightly lower $V_{\text {Grf }}$ appears at $B \sim 2.6 \mathrm{~T}$ and eventually disappears again at $B \sim 4.4 \mathrm{~T}$.

The signal at $B=0 \mathrm{~T}$ arises from tunneling between the anticrossing singlet states $S(1,15)$ and $S(2,14)$ as given by 

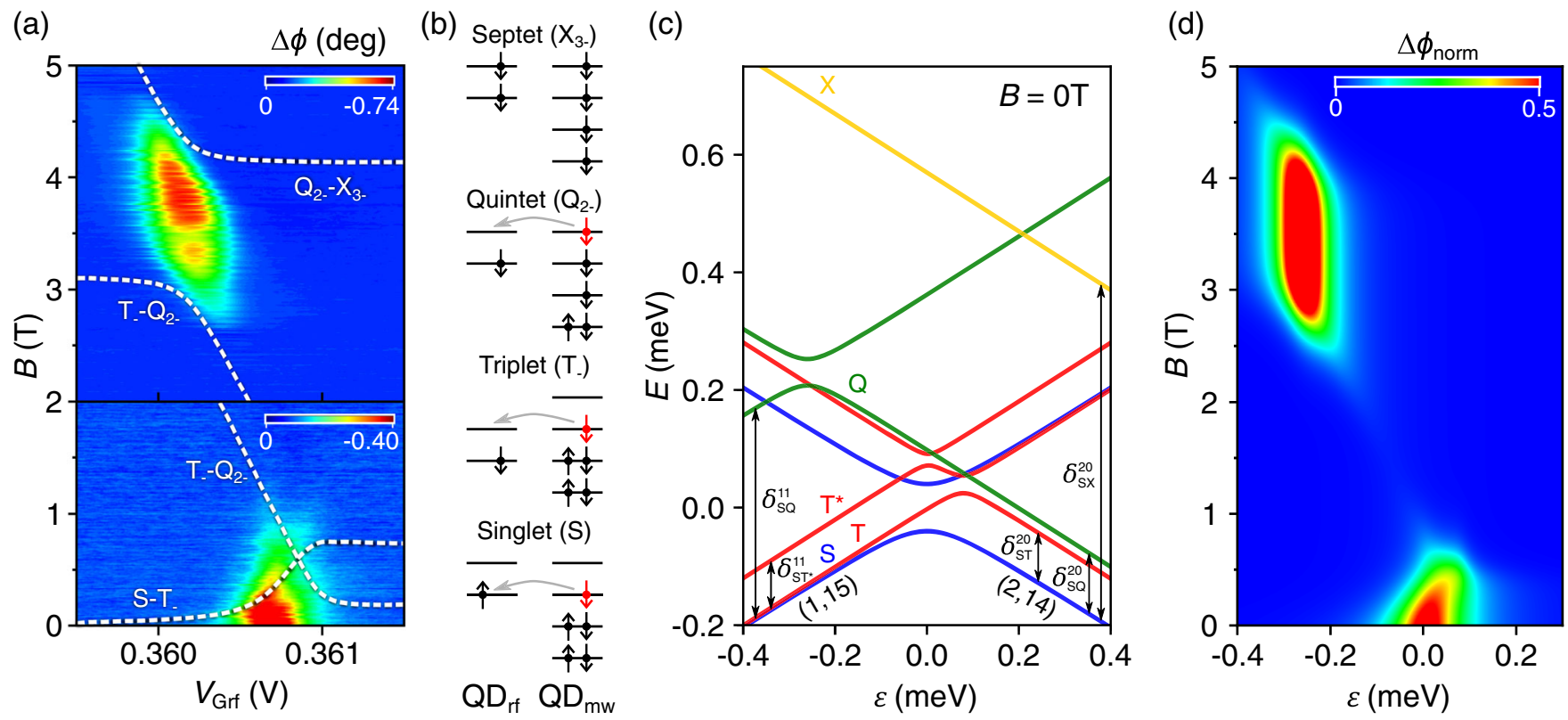

FIG. 2. Pauli spin blockade and energy levels of spin states with $S \leq 3$. (a) Measurement of the $(1,15)-(2,14)$ interdot charge transition at $V_{\mathrm{Gmw}}=0.440 \mathrm{~V}$ as a function of external in-plane magnetic field. From bottom to top, the dashed lines indicate fits to the S-T , $\mathrm{T}_{-}-\mathrm{Q}_{2-}$, and $\mathrm{Q}_{2-}-\mathrm{X}_{3-}$ energy-level intersection points at varying magnetic fields. (b) Illustrative single-particle energy diagrams of the singlet $\mathrm{S}\left(m_{s}=0\right)$, triplet $\mathrm{T}_{-}\left(m_{s}=-1\right)$, quintet $\mathrm{Q}_{2_{-}}\left(m_{s}=-2\right)$, and septet $\mathrm{X}_{3_{-}}\left(m_{s}=-3\right)$ states that successively become the ground state when increasing the magnetic field in panel (a). For simplicity, the energy diagrams omit the five lowest-lying energy levels of $\mathrm{QD}_{\mathrm{mw}}$. The $(1,15)-(2,14)$ anticrossings of the singlet, triplet, and quintet shown in panel (c) are demonstrated by an electron (in red) tunneling from $\mathrm{QD}_{\mathrm{mw}}$ to $\mathrm{QD}_{\mathrm{rf}}$. (c) Energy levels of the double quantum dot simulated based on parameters extracted from panel (a) as a function of $(1,15)-(2,14)$ detuning. The different colors indicate states of varying multiplicity and spin angular momentum $S$ : a singlet with $S=0$ (blue), triplets with $S=1$ (red), quintets with $S=2$ (green), and septets with $S=3$ (yellow). The lines with positive slope are $(1,15)$ states, while lines with negative slope are $(2,14)$ states. These states mix near the anticrossings due to $(1,15)-(2,14)$ interdot tunneling. (d) Normalized phase response as a function of magnetic field simulated based on the energy levels presented in panel (c). In order to enhance the visibility of low-field features, the $z$ scale is limited to half of the normalized phase response.

Eq. (1) and as sketched in the lower panel of Fig. 2(b). The curvature of the anticrossing singlet states is apparent from their calculated eigenenergies that appear in blue in Fig. 2(c) as a function of energy detuning between dots $\varepsilon=$ $e \alpha\left(V_{\mathrm{Grf}}-V_{\mathrm{Grf}}^{0}\right)$, where $V_{\mathrm{Grf}}^{0}=0.3607 \mathrm{~V}$ in this experiment. When $B$ is increased, excited states with a nonzero spin-angular-momentum projection onto the direction of $B$, $m_{s} \neq 0$, will Zeeman split according to $E_{Z}=m_{s} g \mu_{B} B$, where $g$ is the Landé $g$ factor and $\mu_{B}$ is the Bohr magneton. A Zeeman-split state, for example, the triplet $\mathrm{T}_{-}$with $m_{s}=-1$ illustrated in Fig. 2(b), can therefore become the ground state at sufficiently large $B$. In this scenario, the tunneling between $\mathrm{T}(1,1)$ - and $\mathrm{S}(2,0)$-like states- $\mathrm{T}_{-}(1,15)$ and $\mathrm{S}(2,14)$ here-is forbidden by the Pauli exclusion principle [14,39-41], thereby leading to Pauli spin blockade: The ICT signal disappears because $T_{-}(1,15)$ shows no curvature at detuning $\varepsilon=0$ and thus makes no contribution to $C_{\mathrm{Q}}$. One may therefore initially be led to believe that the lack of signal from $B=1.2-2.6 \mathrm{~T}$ in Fig. 2(a) is due to singlet-triplet spin blockade and that the signal at $B=2.6-4.4 \mathrm{~T}$ arises from the curvature of the $\mathrm{T}_{-}(1,15)-\mathrm{T}_{-}(2,14)$ anticrossing $[27,42,43]$. However, as we explain below, this is not the case.
In the lower part of Fig. 2(a), we note that as $B$ is increased from 0 to $0.8 \mathrm{~T}$, the ICT signal decreases in intensity asymmetrically from the left. The reduction of the signal can be ascribed to the lowering of $\mathrm{T}_{-}$below the singlet ground state. This case is illustrated by the dashed white line labeled S-T_ in Fig. 2(a), which tracks the position of the $\mathrm{S}-\mathrm{T}_{-}$crossing above which $\mathrm{T}_{-}$becomes energetically favorable compared to $\mathrm{S}$ as previously explained. This case is also illustrated in Fig. 5 in Appendix B, which shows energy diagrams similar to Fig. 2(c) but at nonzero magnetic fields. Because the signal of the singlet disappears at lower $V_{\text {Grf }}$ first, the anticrossing between $\mathrm{T}_{-}(1,15)$ and $\mathrm{T}_{-}(2,14)$ states must appear at larger gate voltage. For that reason, the signal in the region $B=2.6-4.4 \mathrm{~T}$ cannot arise from triplet tunneling but, as we demonstrate below, instead comes from hybridized quintet spin states $(S=2)$. By closer inspection of the $B=0.8-1.2$ T region (see Fig. 6 of Appendix C), we do, however, identify the signal of the $\mathrm{T}_{-}$anticrossing. This signal overlaps that of the low-field singlet because the singlet-triplet splitting of this system, $\delta_{\mathrm{ST}}$, is small relative to the tunnel coupling of the singlet states, $\Delta_{S}$, thereby making the signals of the $\mathrm{S}$ and $\mathrm{T}_{-}$difficult to discern. The 
triplet signal is accompanied by a second peak (Fig. 6) arising from the tunneling between an excited triplet $\mathrm{T}^{*}(1,15)$ and $\mathrm{T}_{-}(2,14)$ as illustrated with the red eigenenergies in Fig. 2(c). This additional triplet anticrossing explains the extension of the low-field ICT signal beyond the $\mathrm{S}-\mathrm{T}_{-}$crossing line.

As we increase the magnetic field further, the triplet signals vanish at about $B=1.2 \mathrm{~T}$, but at $B=2.6 \mathrm{~T}$, a new signal starts appearing as displayed in the upper part of Fig. 2(a). We attribute this new signal to the anticrossing between the lowest Zeeman-split quintet states $\mathrm{Q}_{2-}(2,14)$ and $\mathrm{Q}_{2-}(1,15)$ with $m_{s}=-2$ [green lines in Fig. 2(c)], which have four electrons aligned with the external magnetic field, as illustrated in Fig. 2(b). Consequently, the $\mathrm{Q}_{2-}$ state experiences twice the Zeeman splitting in comparison to the $\mathrm{T}_{-}$and $\mathrm{T}^{*}{ }_{-}$states, thereby explaining how the quintet state can become a ground state at a sufficiently large magnetic field as shown in Fig. 5(c). Just as the $T_{-}$state blocks the singlet state at lower fields, the low-energy $\mathrm{Q}_{2-}$ state now crosses the $\mathrm{T}_{-}$state as shown with the dashed white line labeled $\mathrm{T}_{-}-\mathrm{Q}_{2-}$ in Fig. 2(a), causing triplet-quintet spin blockade in the $B=1.2-2.6 \mathrm{~T}$ range. We note that the discontinuity of the fitted dashed $\mathrm{T}_{-}-\mathrm{Q}_{2-}$ line at $B=2 \mathrm{~T}$ may be due to changes in charge in the peripheral environment of the DQD between measurements. Increasing the magnetic field beyond $B=4.4 \mathrm{~T}$ results in a six-electron $\mathrm{X}_{3-}$ septet state with $S=3$ and $m_{s}=-3$ [top panel of Fig. 2(b) and yellow line in Fig. 2(c)], which experiences thrice the Zeeman splitting compared to the triplet, to move below the $\mathrm{Q}_{2}$ state and become the new ground state as shown in Fig. 5(d). This result generates yet another region of spin blockade as seen in the upper part of Fig. 2(a). Magnetospectroscopy measurements with features similar to those presented here were obtained for a neighboring even-parity ICT with two fewer electrons on $\mathrm{QD}_{\mathrm{mw}}$ as well as in another similar device (see Sec. S1 of the Supplemental Material [44]).

From the measurements presented in Fig. 2(a), we compile quantitative information about the energy spectrum of the DQD, which we use to produce Fig. 2(c), showing the singlet (blue), triplet (red), quintet (green), and septet (yellow) states at $B=0 \mathrm{~T}$. We obtain $\alpha=0.345$ from the slopes of the asymmetrically vanishing ICT signals [dashed lines in Fig. 2(a)]. We get the minimum energy separation between states with the same total spin number-the tunnel couplings-from the full width at half maximum (FWHM) of the signals at a fixed $B$ and thus extract $\Delta_{\mathrm{S}}=80 \mu \mathrm{eV}$ for the singlet at $B=0 \mathrm{~T}$ and $\Delta_{\mathrm{Q}}=45 \mu \mathrm{eV}$ for the quintet at $B=3.71 \mathrm{~T}$ and estimate $\Delta_{\mathrm{T}}=35 \mu \mathrm{eV}$ and $\Delta_{\mathrm{T}^{*}}=20 \mu \mathrm{eV}$ at $B=1.02 \mathrm{~T}$ for the $\mathrm{T}_{-}(1,15)-\mathrm{T}_{-}(2,14)$ and the $\mathrm{T}_{-}^{*}(1,15)-\mathrm{T}_{-}(2,14)$ tunnel couplings, respectively (the method for extracting the tunnel couplings is described in Appendix D). Finally, from the signal position on the $V_{\text {Grf }}$ axis and the magnetic fields at which the different spin blockades occur, we extract the energy splitting between states in both the $(2,14)$ and the $(1,15)$ configurations, which, for simplicity, we refer to as $(2,0)$ and $(1,1)$, respectively. We get the singlettriplet splitting on the $(2,0)$ side, $\delta_{\mathrm{ST}}^{20}=80 \mu \mathrm{eV}$; the singlettriplet* splitting on the $(1,1)$ side, $\delta_{\mathrm{ST}^{*}}^{11}=80 \mu \mathrm{eV}$; the singlet-quintet splitting on the $(1,1)$ side, $\delta_{\mathrm{SQ}}^{11}=360 \mu \mathrm{eV}$, and the $(2,0)$ side, $\delta_{\mathrm{SQ}}^{20}=100 \mu \mathrm{eV}$; and lastly, the singletseptet splitting on the $(2,0)$ side, $\delta_{\mathrm{SX}}^{20}=570 \mu \mathrm{eV}$. The smallest splittings, $\delta_{\mathrm{ST}}^{20}$ and $\delta_{\mathrm{ST}^{*}}^{11}$, may be associated with valley splittings, while the others involve combinations of valley and orbital excitations in $\mathrm{QD}_{\mathrm{mw}}$. The relatively small $\delta_{\mathrm{ST}}^{20}$, which is associated with $\mathrm{QD}_{\mathrm{rf}}$, is in the lower range of values reported for this class of devices (few tens to few hundreds of $\mu \mathrm{eV}$ [45-49]), which may be linked to a weak vertical electrical field and to the condition of the $\mathrm{Si} / \mathrm{SiO}_{2}$ interface. We note that the valley splitting may be enhanced by applying a negative back-gate voltage [47] or by reducing the number of electrons in the DQD, which strengthens vertical confinement.

In the context of spin-qubit implementation, it is important to observe that splittings of high-spin states, e.g., $\delta_{\mathrm{SQ}}^{20}$ and $\delta_{\mathrm{SQ}}^{11}$, comparable to or smaller than the high-spin Zeeman splitting at the magnetic field of operation, may compromise manipulation and readout of single-spin and singlet-triplet qubits, as is explained in Appendix E. These considerations are particularly relevant to multielectron planar and corner DQDs as they may be more likely to exhibit an increased number of near-degenerate excited states [11].

Using the parameters introduced above, we build the multispin Hamiltonian of the system as described in Appendix $\mathrm{F}$ and extract the eigenenergies $E_{i}$ as plotted in Figs. 2(c) and 5. Using the expression for $C_{\mathrm{Q}}$ in Eq. (1) and the magnetic field dependence of the eigenenergies, we then obtain the simulated magnetospectroscopy map in Fig. 2(d) for which we included a finite electron temperature $T=175 \mathrm{mK}$. The good match between data and simulation confirms our understanding of the DQD energy spectrum and thereby allows for the possibility of probing the dynamical properties of the high-spin states accessible in this DQD.

\section{RELAXATION OF THE QUINTET STATE}

Spin relaxation time $T_{1}$ is an important metric as it ultimately limits coherence and determines the minimum readout time required for achieving high-fidelity spin readout [24]. Spin relaxation in silicon has been measured for single spins [20], singlet-triplet systems [41], hybrid qubits [50], and hole spins $[10,16]$. In the following, we explore spin relaxation from spin-quintet states. To determine the relaxation time of the $\mathrm{Q}_{2-}$ state, we first set $B=2.5 \mathrm{~T}$, at which point the $\mathrm{DQD}$ is under triplet-quintet spin blockade. Figure 3(a) shows the energy levels at $B=2.5 \mathrm{~T}$ as simulated based on the parameters extracted from magnetospectroscopy. At this field, the $\mathrm{Q}_{2-}$ 
(a)

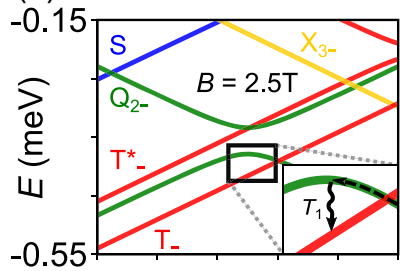

(c)

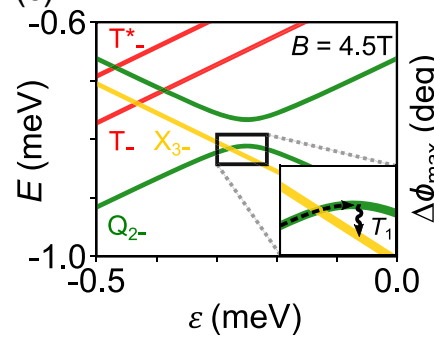

(b)

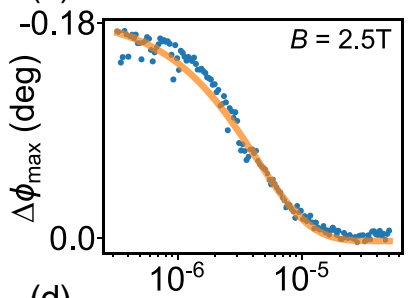

(d)

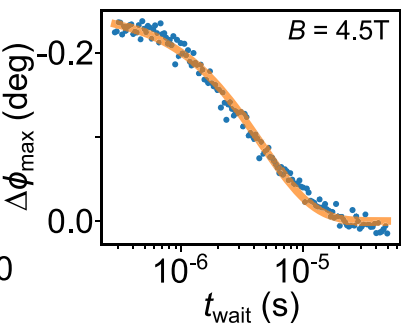

FIG. 3. Quintet relaxation. (a,c) Energy levels of the double quantum dots at magnetic fields of $B=2.5 \mathrm{~T}$ and $B=4.5 \mathrm{~T}$ simulated based on parameters extracted from magnetospectroscopy measurements presented in Fig. 2. The insets highlight the pulsing into the quintet $\mathrm{Q}_{2-}$ anticrossing and the subsequent relaxation into the triplet $\mathrm{T}_{-}$and septet $\mathrm{X}_{3-}$ ground states, respectively. (b,d) Decay of the maximum ICT phase response $\Delta \phi_{\max }$ as a function of wait time $t_{\text {wait }}$ at the $\mathrm{Q}_{2-}$ anticrossing. By fitting to a single exponential decay (orange line), we observe quintet relaxation times of $T_{1}^{\mathrm{Q} \rightarrow \mathrm{T}}=4.26 \pm 0.11 \mu \mathrm{s}$ and $T_{1}^{\mathrm{Q} \rightarrow \mathrm{X}}=4.59 \pm 0.11 \mu \mathrm{s}$.

anticrossing at $\varepsilon \sim-0.25 \mathrm{meV}$ lies at energies slightly higher than the energy of the $\mathrm{T}_{-}$state; however, at sufficiently large $\varepsilon, \mathrm{Q}_{2-}(2,14)$ is the ground state. To probe the $\mathrm{Q}_{2-}$ anticrossing, we therefore apply a 50\%-dutycycle square-wave voltage pulse to $\mathrm{G}_{\mathrm{mw}}$ that is initialized (I) from the $(1,14)$ configuration and then pulsed to the measurement point $(\mathrm{M})$ on the ICT as seen in the inset of Fig. 1(b). When crossing the $(1,14)-(2,14)$ charge transition, an electron is loaded into $\mathrm{QD}_{\mathrm{rf}}$ at a rate $\Gamma \gtrsim f_{\mathrm{rf}}$, given that the transition produces a measurable phase shift in the resonator response [51]. We set the rise and fall time of the pulse to $10 \mathrm{~ns}$, much slower than the tunneling time, such that the system is initialized in the energetically favored $\mathrm{Q}_{2-}(2,14)$ state. The quintet state is subsequently followed through adiabatic passage to the $\mathrm{Q}_{2-}$ anticrossing (point M), as illustrated in the inset of Fig. 3(a), causing a nonthermal occupation probability. Finally, the electron is unloaded and the system reset via the reverse procedure.

To measure $T_{1}$, we wait for a time $t_{\text {wait }}$ at point $\mathrm{M}$, during which the $\mathrm{Q}_{2-}$ state relaxes to the $\mathrm{T}_{-}$state with a characteristic relaxation time $T_{1}$ as indicated in the inset of Fig. 3(a). When spending very little time $\left(t_{\text {wait }} \ll T_{1}\right)$ at the measurement point of the pulse sequence, the probability that the $\mathrm{Q}_{2-}$ state relaxes tends to 0 , which leads to lifting of the spin blockade and regeneration of the ICT phase shift signal from the curvature of the $\mathrm{Q}_{2-}$ state according to Eq. (1). Contrarily, if $t_{\text {wait }} \gg T_{1}$, the probability that the

$\mathrm{Q}_{2-}$ state relaxes to the noncurving $\mathrm{T}_{-}$state tends to 1 , and we therefore no longer measure the ICT signal, which is averaged over the duration of the pulse.

We repeat the pulse sequence described above $N>$ $2.4 \times 10^{5}$ times for various $t_{\text {wait }}$ and with the rf drive continuously on, thus measuring the average $\Delta \phi$ of the voltage region around the $\mathrm{Q}_{2-}$ anticrossing. From fitting the $\Delta \phi$ line trace to $\Delta \phi \propto c_{1}\left[\left(\varepsilon-\varepsilon_{0}\right)^{2}+\left(\Delta_{\mathrm{Q}}\right)^{2}\right]^{-3 / 2}+c_{2}$, where $\varepsilon_{0}$ and $\Delta_{\mathrm{Q}}$ are the detuning and the tunnel coupling of the quintet anticrossing, respectively, and $c_{i}$ are constants [36], the maximum phase shift $\Delta \phi_{\max }$ is extracted. In order to obtain the $\mathrm{Q}_{2-}-\mathrm{T}_{-}$relaxation time, $\Delta \phi_{\max }$ is then plotted as a function of $t_{\text {wait }}$ and fitted to a single exponential decay, $A_{1} e^{-t_{\text {wait }} / T_{1}^{\mathrm{Q} \rightarrow \mathrm{T}}}+A_{2}$, where $A_{i}$ are constants, as shown in Fig. 3(b), whereby we find $T_{1}^{\mathrm{Q} \rightarrow \mathrm{T}}=4.26 \pm 0.11 \mu \mathrm{s}$.

Setting the magnetic field to $B=4.5 \mathrm{~T}$, we now study the quintet in the region of quintet-septet spin blockade, illustrated in Fig. 3(c), which shows the simulated energy levels at this magnetic field. While similar to the lower-field triplet-quintet scenario, here the $\mathrm{Q}_{2-}$ state is the ground state at $\varepsilon \lesssim-0.25 \mathrm{meV}$, i.e., in the $(1,15)$ configuration, rather than at $\varepsilon \gtrsim-0.25 \mathrm{meV}$. This change affects the pulsing scheme required to determine the relaxation time. The voltage pulses on $\mathrm{G}_{\mathrm{mw}}$ are modified accordingly, starting instead from the $(2,15)$ state $\left(\mathrm{I}^{\prime}\right)$ in order to initialize the system in the energetically favored $\mathrm{Q}_{2-}(1,15)$ before moving via adiabatic passage to the measurement point $\left(\mathrm{M}^{\prime}\right)$ as shown in the insets of Figs. 1(b) and 3(c). As in the quintet-triplet case, $\Delta \phi_{\max }$ is extracted from the ICT phase response and fitted against a single exponential decay, $A_{3} e^{-t_{\text {wait }} / T_{1}^{\mathrm{Q} \rightarrow \mathrm{X}}}+A_{4}$, shown in orange in Fig. 3(d), which results in a $\mathrm{Q}_{2-}-\mathrm{X}_{3-}$ relaxation time $T_{1}^{\mathrm{Q} \rightarrow \mathrm{X}}=4.59 \pm$ $0.11 \mu \mathrm{s}$ that is comparable to $T_{1}^{\mathrm{Q} \rightarrow \mathrm{T}}$. While there are no other reported quintet relaxation timescales to benchmark these results to, it is about 3 orders of magnitude smaller than previously reported relaxation times in singlet-triplet DQD systems $[41,52,53]$. The fast quintet relaxation calls for the existence of a spin mixing mechanism such as spinorbit coupling.

Recent studies of singlet-triplet relaxation in a silicon DQD reveal that the lifetime decreases monotonically with decreasing $\mathrm{S}(0,2)-\mathrm{T}_{-}(1,1)$ splitting [52], which suggests enhanced singlet-triplet mixing near the degeneracy point. In silicon, the additional valley degree of freedom gives rise to a general spin-valley-orbit coupling mechanism, and in low-symmetry quantum dots, such as the corner dots presented here, there is evidence that this mechanism can be more pronounced than in conventional planar quantum dots or dopants [46]. The degree of spin mixing also depends on how close the energies of the different states are. Since $\mathrm{QD}_{\mathrm{mw}}$ hosts a large number of electrons-up to 15 in this case-the excited-state energy spectrum is quite dense around the Fermi energy, as assessed experimentally, which multiplies the opportunities for spin-valley-orbit mixing. 
A likely hypothesis for the significantly shorter quintet relaxation time is therefore an enhanced spin-orbit mixing within a dense energy spectrum. This hypothesis is supported by Ref. [11], which shows that the relaxation rate can be highly dependent on the filling and level of confinement of the dots, in particular, in highly excited shells with increased multiplicity.

In consideration of the aforementioned effects, we suggest studying high-spin states in quantum dots with reduced electron occupancy $(N=4,6$ for quintets and septets) and with tunable vertical electric fields (via top or back gating) in order to obtain a more accurate representation of the high-spin relaxation time.

\section{CONCLUSIONS AND OUTLOOK}

In summary, we have demonstrated the formation of a tunnel-coupled DQD in the channel of a split-gate silicon transistor. By embedding the device in an LC electrical resonator and performing gate-based dispersive sensing, we have determined the charge state of the DQD down to the last electron. By tuning to the $(1,15)-(2,14)$ interdot charge transition and studying the quantum capacitance of the system as a function of the magnetic field, we have found evidence of multiparticle high-spin states not studied before, namely, electron spin quintets $(S=2)$ and spin septets $(S=3)$. We attribute the appearance of these high-spin states in the DQD to a dense excited-state spectrum, which may be of particular relevance to multielectron planar and corner silicon quantum dots. From the magnetospectroscopy measurements, we determined the energy spectrum as a function of energy detuning between dots, as well as the coupling energy between states with equal spin numbers. Additionally, we have developed a model to describe the magnetic field dependence of the dispersive response based on the multistate quantum capacitance of the system. The model reproduces the experimental results well and generally provides a tool for understanding the dispersive response of systems with a complex spin configuration. Finally, nonequilibrium studies measured under spin blockade and presented here demonstrate a typical quintet relaxation time, $T_{1} \sim 4 \mu \mathrm{s}$, at the hybridization point. Overall, our results provide a way to reconstruct the energy spectrum of complex spin systems and allow for the possibility of using CMOS quantum dots as a tunable test bed for studying the interactions and dynamics of high-spin systems.

\section{ACKNOWLEDGMENTS}

This research has received funding from the European Union's Horizon 2020 Research and Innovation Programme under Grant Agreement No. 688539 (Ref. [54]). T. L. acknowledges support from the EPSRC Cambridge NanoDTC, EP/L015978/1. M. F. G.Z. acknowledges support from the Royal Society and the Winton Programme for the Physics of Sustainability. Y. M.
N. and J. L. acknowledge support from the French National Research Agency (ANR project MAQSi). D. J. N. acknowledges the French National Research Agency under the programme Investissements davenir' and the GreQuE doctoral programmes (ANR-15-IDEX-02 and Grant Agreement No. 754303). This work was supported by the French National Research Agency through the CODAQ project (ANR-16-ACHN-0029).

\section{APPENDIX A: CHARGE STABILITY DIAGRAM IN THE FEW-ELECTRON REGIME}

Figure 4 presents the same data as Fig. 1(b) but with no inset and with a zoom-in on the few-electron region. By close inspection of the quasihorizontal (quasivertical) lines that represent $\mathrm{QD}_{\mathrm{mw}}$-to-reservoir $\left(\mathrm{QD}_{\mathrm{rf}}\right.$-to-reservoir)
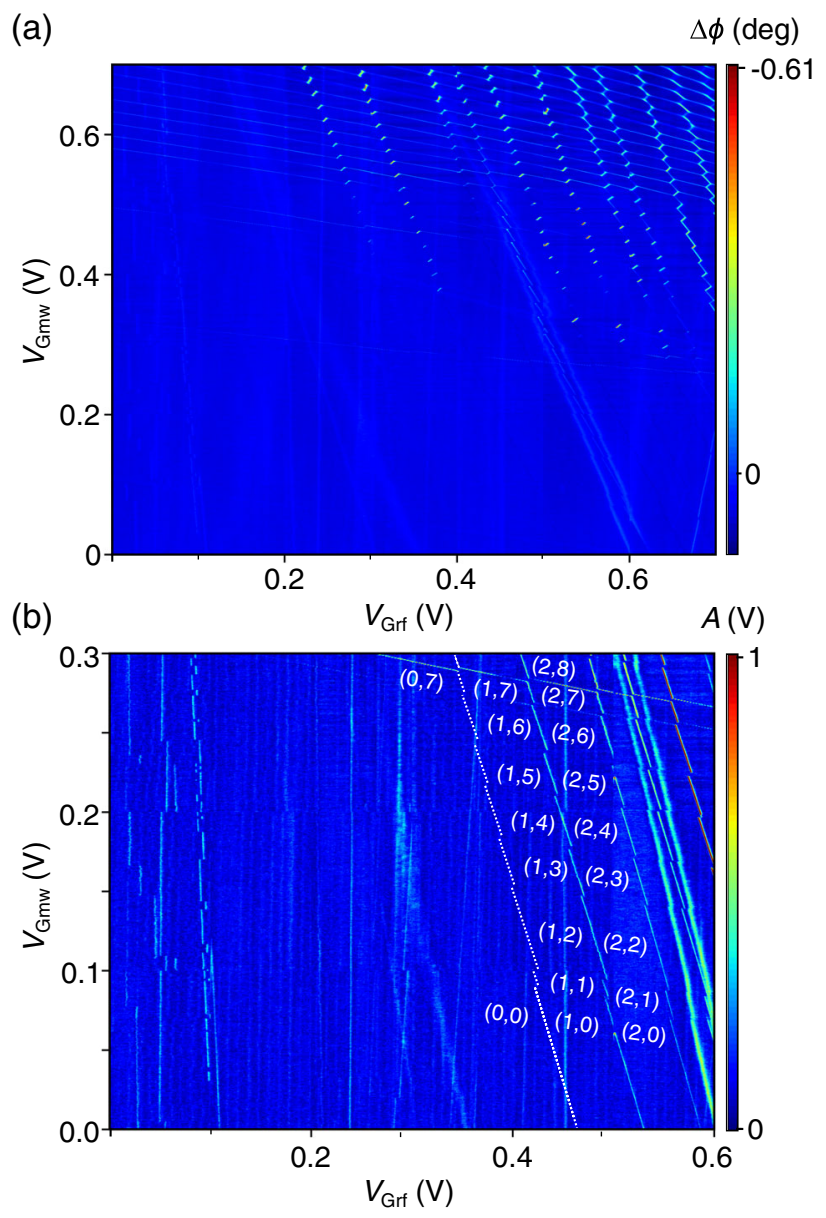

FIG. 4. Charge stability diagrams of the DQD recorded using dispersive gate-based readout, where panel (a) is similar to Fig. 1(b) without the inset and panel (b) is a zoom-in on the few-electron regime showing the amplitude $A$ instead of $\Delta \phi$ of the IQ demodulated response. The dotted white line indicates the addition of the first electron to $\mathrm{QD}_{\mathrm{rf}}$, its location determined by mimicking the $(1, n)-(2, n)$ line and placing it at the position of the first visible ICT involving zero electrons in $\mathrm{QD}_{\text {rf }}$, i.e., $(0,8)$ $(1,7)$. Panels (a) and (b) are stitched together by 49 and 18 individual measurements, respectively. 
transitions, it is possible to observe kinks that, due to the capacitive coupling between the QDs, indicate the addition of one electron to $\mathrm{QD}_{\mathrm{rf}}\left(\mathrm{QD}_{\mathrm{mw}}\right)$. In other words, we use the dispersive signal of one QD exchanging electrons with a reservoir as a sensor for detecting the charge of the other QD. Thus, by counting the number of kinks, the electron occupancy of the DQD can therefore be established as a function of gate voltage. This is illustrated in Fig. 4(b), where $i(j)$ in $(i, j)$ represents the charge occupancy of $\mathrm{QD}_{\mathrm{rf}}\left(\mathrm{QD}_{\mathrm{mw}}\right)$. The kinks in the charge stability diagram are not to be confused with the discontinuities along vertical and horizontal lines that are multiples of $0.1 \mathrm{~V}$, e.g., at $\left(V_{\mathrm{Grf}}, \mathrm{V}_{\mathrm{Gmw}}\right)=(0.5,0.063)$, as these are due to stitching of individual measurements, each $0.1 \mathrm{~V} \times 0.1 \mathrm{~V}$ in size. (a)

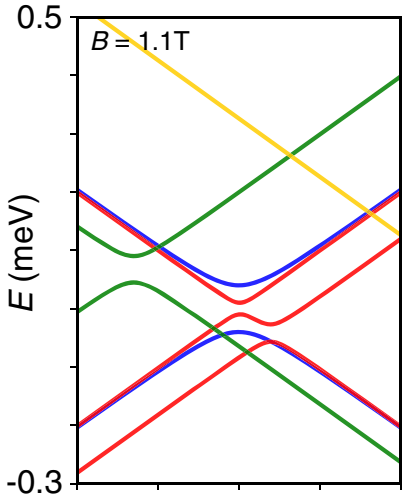

(c)

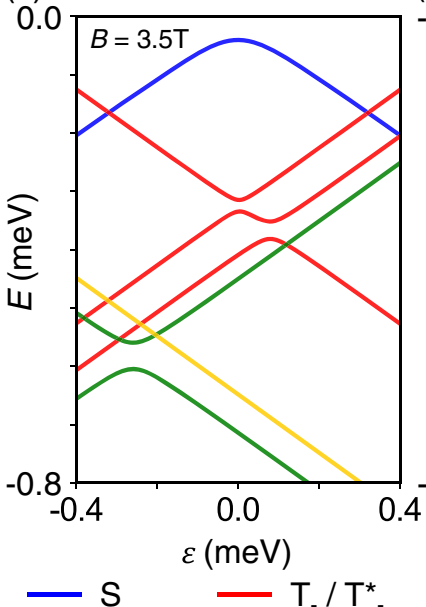

(b)

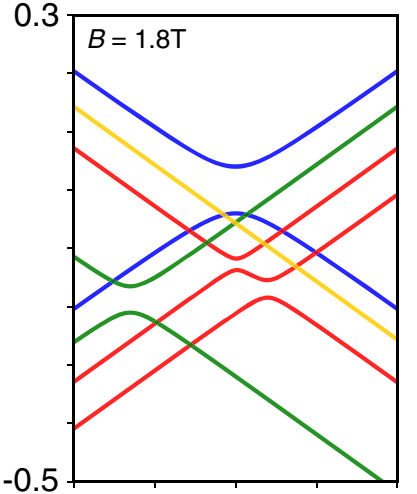

(d)

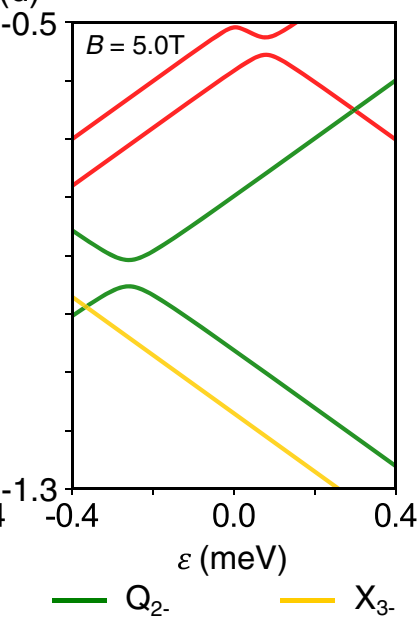

FIG. 5. Energy levels of the lowest-energy spin state of each spin manifold with $S \leq 3$ at various magnetic fields as a function of detuning $\varepsilon$. The singlet states (S) are shown in blue, the triplet states $\left(\mathrm{T}_{-}\right)$in red, the quintet states $\left(\mathrm{Q}_{2-}\right)$ in green, and the septet state $\left(\mathrm{X}_{3_{-}}\right)$in yellow. Each panel shows the energy spectrum at the magnetic field indicated in the upper left of the panel and has the following physical relevance for the system: (a) Onset of quintet-triplet spin blockade and origin of double peak in Fig. 6. (b) Quintet-triplet spin blockade. (c) Lifting of spin blockade as the quintet anticrossing becomes the ground state. (d) Septetquintet spin blockade.

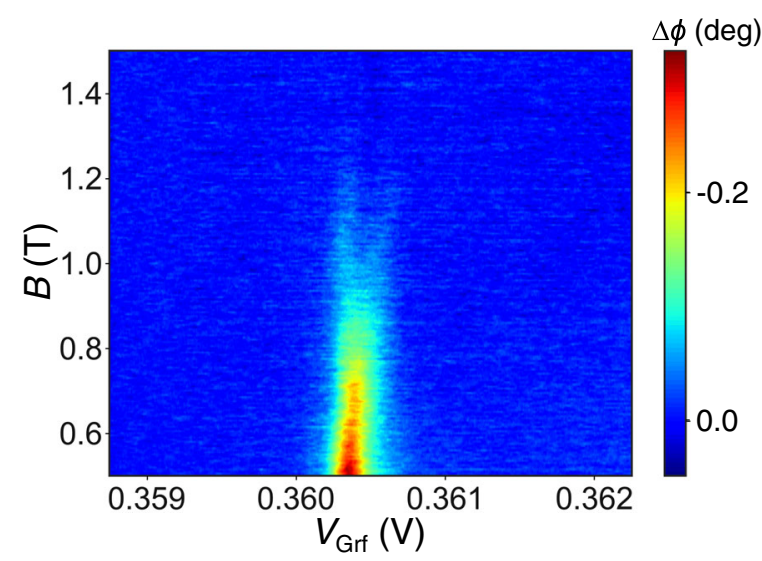

FIG. 6. Dispersive measurement of the $(1,15)-(2,14)$ interdot charge transition at $V_{\mathrm{Gmw}}=0.440 \mathrm{~V}$ as a function of external inplane magnetic field from $B=0.5 \mathrm{~T}$ to $1.5 \mathrm{~T}$. In the $B=0.9 \mathrm{~T}$ to $1.2 \mathrm{~T}$ region, a double peak arising from the tunneling between triplet states, $\mathrm{T}_{-}$and $\mathrm{T}^{*}$, is visible.

\section{APPENDIX B: MANY-PARTICLE ENERGY DIAGRAMS AT VARIOUS MAGNETIC FIELDS}

Figure 5 plots the eigenenergies of the lowest-energy $S \leq 3$ spin states similar to Fig. 2(c) but here at various nonzero magnetic fields (for simplicity, we include only the lowest energy state of each spin manifold). Figure 5(a) shows the energies at $B=1.1 \mathrm{~T}$, a point at which the $\mathrm{T}_{-}$states (red) are energetically favorable compared to the singlet state (blue) and give rise to the double-peak signal in Figs. 6 and 7(c). We note that the $\mathrm{Q}_{2-}$ state (green) already blocks both the singlet and triplet states, which explains the weak intensity of the phase response signal at this magnetic field. At $B=1.8 \mathrm{~T}$ in Fig. 5(b), the triplets are fully blocked, and this panel thus illustrates the energy spectrum under quintet-triplet spin blockade. Figure 5(c) shows a magnetic field, $B=3.5 \mathrm{~T}$, where the anticrossing $\mathrm{Q}_{2-}$ states are now the ground state, thus leading to lifting of the spin blockade. Finally, at $B=5.0 \mathrm{~T}$, the septet $\mathrm{X}_{3-}$ state is energetically favorable compared to the $\mathrm{Q}_{2-}$ state, thereby leading to septet-quintet spin blockade.

\section{APPENDIX C: MAGNETOSPECTROSCOPY OF THE LOW MAGNETIC FIELD REGION}

Figure 6 presents a high-resolution magnetospectroscopy measurement of the $(1,15)-(2,14)$ interdot charge transition at $V_{\mathrm{Gmw}}=0.440 \mathrm{~V}$ as a function of external inplane magnetic field focused on the region from $B=0.5 \mathrm{~T}$ to $B=1.5 \mathrm{~T}$. The dispersive signal at $B=0.5 \mathrm{~T}$ arises from the singlet states as described in Sec. III. It disappears asymmetrically from the left and is superseded by the signal from the $\mathrm{T}_{-}$and $\mathrm{T}^{*}$ - anticrossings at around $B=0.9 \mathrm{~T}$, which are shown in red in Figs. 2(c) and 5 . The left part of the double peak is attributed to the 
$\mathrm{T}^{*}(1,15)-\mathrm{T}_{-}(2,14)$ anticrossing, whereas the right part is attributed to the $\mathrm{T}_{-}(1,15)-\mathrm{T}_{-}(2,14)$ anticrossing.

\section{APPENDIX D: METHOD FOR EXTRACTING THE TUNNEL COUPLING}

In this Appendix, we describe the protocol for extracting the singlet, quintet, and triplet tunnel couplings quoted in Sec. III. For an isolated coupled two-level system, the equation that describes the quantum capacitance line shape as a function of gate voltage is [36]

$$
C_{\mathrm{Q}}=C_{0} \frac{\Delta_{i}^{3}}{\Delta E\left(V_{\mathrm{Grf}}\right)^{3}} \tanh \left[\frac{\Delta E\left(V_{\mathrm{Grf}}\right)}{2 k_{\mathrm{B}} T}\right],
$$

where $C_{0}=(\alpha e)^{2} / 2 \Delta_{i}, \Delta E\left(V_{\mathrm{Grf}}\right)=\left(\alpha^{2}\left(V_{\mathrm{Grf}}-V_{\mathrm{Grf}, \mathrm{i}}^{0}\right)^{2}+\right.$ $\left.\Delta_{i}^{2}\right)^{-\frac{1}{2}}, \Delta_{i}$ is the tunnel coupling of the spin branch $i$, and $V_{\mathrm{Grf}, \mathrm{i}}^{0}$ is the gate voltage corresponding to zero detuning for spin branch $i$. Furthermore, we recall the relation

$$
\Delta \phi=-\frac{2 Q_{1} C_{\mathrm{Q}}}{C_{\mathrm{T}}},
$$

where $Q_{1}$ is the loaded quality factor of the resonator and $C_{\mathrm{T}}$ is the total capacitance of the system. To extract the singlet tunnel coupling $\Delta_{\mathrm{S}}$, the quintet tunnel coupling $\Delta_{\mathrm{Q}}$, and the tunnel coupling between the $\mathrm{T}(1,15)[\mathrm{T} *(1,15)]$ and $\mathrm{T}(2,14)$ triplet states $\Delta_{\mathrm{T}}\left[\Delta_{\mathrm{T}^{*}}\right]$, we use $\Delta \phi$ vs $V_{\text {Grf }}$ traces at $V_{\mathrm{Gmw}}=0.440 \mathrm{~V}$ and at $B=0,3.71$ and $1.02 \mathrm{~T}$, respectively (see Fig. 7). For the fits to the data, we use Eqs. (D1) and (D2) as well as $\alpha=0.345$, extracted from the slopes of the asymmetrically vanishing ICT signals [dashed lines in Fig. 2(a)], and $T=175 \mathrm{mK}$, obtained from the comparison between data and simulations in Figs. 2(a) and 2(d). The fits allow us to extract $\Delta_{i}$, which is directly related to the FWHM of the peaks in Fig. 7.

In the case of the singlet branch [Fig. 7(a)], the symmetry of the trough and the good fit to Eq. (D1) indicate that the assumption of an isolated coupled two-level system holds. From the fit, we obtain $\Delta_{\mathrm{S}}=80 \mu \mathrm{eV}$. Given the large ratio $\Delta_{\mathrm{S}} / k_{\mathrm{B}} T>5$, the triplet states, closest in energy to the (a)

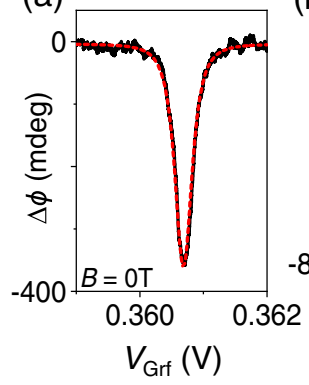

(b)

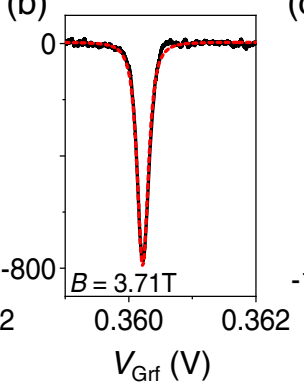

(c)

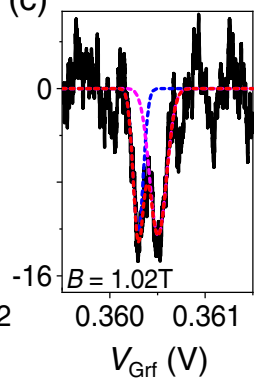

FIG. 7. Phase response as a function of $V_{\text {Grf }}$ at three magnetic fields. The dashed lines indicate fits to the data enabling the determination of $\Delta_{i}$. (a)-(c) Singlet, quintet, and triplet signals, respectively. singlet ground state [see Figs. 2(b) and 2(c)], are largely unpopulated in the detuning region with nonzero energy curvature of the singlet, and the measured signal hence arises predominantly from the singlet ground-state branch.

For the quintet branch [Fig. 7(b)], the symmetry of the trough again indicates an energetically isolated, coupled two-level system. Looking at Fig. 5(c), which shows simulated energy levels as a function of detuning at $B=3.5 \mathrm{~T}$, we verify that this is indeed the case for the hybridized quintets. From the fit, we obtain $\Delta_{\mathrm{Q}}=45 \mu \mathrm{eV}$.

Finally, considering now the triplet branch and measurement at $B=1.02$ T [Fig. 7(c)], we note from Fig. 5(a) that the triplet branch is not the ground state of the system close to $\varepsilon=0$ at this magnetic field. Only a small thermal population of the triplets is therefore responsible for the measured quantum capacitance signal, which also explains the smaller $\Delta \phi$ amplitude of the troughs in Fig. 7(c). In this case, the assumption of an energetically isolated two-level system breaks down, but the linewidth of the peak is still determined by the tunnel coupling to first order, thus allowing estimation of $\Delta_{\mathrm{T}}=35 \mu \mathrm{eV}$ (purple dashed line) and $\Delta_{\mathrm{T}^{*}}=20 \mu \mathrm{eV}$ (blue dashed line) [36].

\section{APPENDIX E: EFFECT OF HIGH-SPIN STATES ON SINGLE SPIN AND SINGLET-TRIPLET QUBIT IMPLEMENTATION}

Figure 2(c) plots the energy spectrum of the double quantum dot as a function of $(1,15)-(2,14)$ detuning and indicates the splitting $\delta$ between states of various spin angular momenta. Given the density of the excited-state energy spectrum, one may consider how high-spin states can affect the implementation of single-spin and singlet-triplet qubits in double quantum dots with such energy spectra. In the following, we assume a single-spin qubit implementation that employs one quantum dot for the qubit and the other as an ancilla dot for readout, in which case the relevant qubit states are $|\downarrow \downarrow\rangle$ and $|\uparrow \downarrow\rangle$ [the reader may find it helpful to refer to the energy diagram of Fig. 2(a) in Ref. [23] ].

We first consider the manipulation stage of single-spin and singlet-triplet qubits, during which the qubit is operated in the $(1,1)$ charge configuration. Here, the splitting between the singlet and the $\mathrm{Q}_{2-}$ quintet branch is $\delta_{\mathrm{SQ}_{2-}}^{11}(B)=\delta_{\mathrm{SQ}}^{11}-2 g \mu_{B} B$, where $\delta_{\mathrm{SQ}}^{11}$ is the singlet-quintet splitting at $B=0$ and the factor of 2 in the Zeeman splitting comes from the magnetic spin number of the $\mathrm{Q}_{2-}$ state. Qubit manipulation may be compromised if the quintet $\mathrm{Q}_{2-}$ state ends at energies similar to or lower than the qubit states as a result of the applied magnetic field. The critical magnetic field at which this happens is given by $B_{c}^{m}=$ $\delta_{\mathrm{SQ}}^{11} /\left(\eta_{m} g \mu_{B}\right)$, where $\eta_{m}$ indicates the difference in Zeeman splitting between the upper qubit state and the $\mathrm{Q}_{2-}$ state; hence, $\eta_{m}=2$ for $\mathrm{ST}_{0}$ qubits and single-spin qubits. It is important to note that for singlet-triplet qubits, the magnitude of the magnetic field need only be on the order of a 
few $\mathrm{mT}$ [40], which is unlikely to make the above considerations experimentally relevant. However, in single-spin qubits, which require magnetic fields of about $1 \mathrm{~T}$ in order not to sacrifice coherence time [23], the above considerations are important.

Next, we consider the impact of high-spin states on single-spin and singlet-triplet qubit readout done via Pauli spin blockade. In the case of readout with charge sensing, the $\mathrm{Q}_{2}$ - state may compromise readout by lifting the triplet spin blockade at the chosen readout point $\varepsilon_{r}$, which lies in the $(2,0)$ charge configuration, i.e., $\varepsilon_{r}>0$. Triplet spin blockade may be lifted for magnetic fields larger than the critical magnetic field for readout $B_{c}^{r}$, which is given by the condition $E_{\mathrm{Q} 2-}\left(B_{c}^{r}, \varepsilon_{r}\right)=E_{|\downarrow \downarrow\rangle}\left(B_{c}^{r}, \varepsilon_{r}\right)$ for single-spin qubits and $E_{\mathrm{Q}_{2}-}\left(B_{c}^{r}, \varepsilon_{r}\right)=E_{\mathrm{T}_{0}}\left(\varepsilon_{r}\right)$ for singlet-triplet qubits. Solving for $B_{c}^{r}$ in the conditions above yields

$$
B_{c}^{r}=\frac{3 \delta_{\mathrm{SQ}}^{20}-2 \delta_{\mathrm{ST}}^{20}-\varepsilon_{r}+\sqrt{\left(\varepsilon_{r}-\delta_{\mathrm{SQ}}^{20}\right)^{2}+\Delta_{\mathrm{T}}^{2}}}{2 \eta_{r} g \mu_{B}},
$$

assuming $B_{c}^{r}>\left(\delta_{\mathrm{SQ}}^{20}-\delta_{\mathrm{ST}}^{20}\right) /\left(\eta_{r} g \mu_{B}\right)$, which is required for spin-blockade lifting and where $\eta_{r}=1$ for single-spin qubits and $\eta_{r}=2$ for singlet-triplet qubits. If $B>B_{c}^{r}$, the point of spin-blockade lifting, $\varepsilon_{l}$, will occur at a detuning smaller than the readout point, $\varepsilon_{r}>\varepsilon_{l}>0$, and if the readout integration time is also comparable to or longer than $T_{1}^{|\downarrow \downarrow\rangle \rightarrow \mathrm{Q}}$ for single-spin qubits and $T_{1}^{\mathrm{T}_{0} \rightarrow \mathrm{Q}}$ for singlet-triplet qubits, charge sensing-based readout fidelity will be negatively affected.

In the case of dispersive readout, the readout mechanism relies on the fact that only the singlet produces a nonzero phase response at $\varepsilon=0$, as described in the main text. If a quintet state gets close in energy to the singlet state, i.e., $\Delta_{\mathrm{S}} / 2+\delta_{\mathrm{SQ}}^{20}-2 g \mu_{B} B<4 k_{B} T$, and $T_{1}^{\mathrm{S} \rightarrow \mathrm{Q}}$ is comparable to or shorter than the readout time, a proportion of the singlet population will thermalize and populate the quintet state, which generates no signal at $\varepsilon=0$. This will reduce the singlet signal strength and thus the readout fidelity. As in the case of qubit manipulation described above, single-spin qubit implementations will suffer more from these readout considerations given the relatively larger magnetic field required. Additionally, we note that in order to maximize the readout window in the $Z$ valleys, the DQD may be operated at a $(3,1)-(4,0)$ transition, where the lowest-lying excitation in the $(4,0)$ readout state is an orbital splitting and therefore presumably larger than the valley splitting [41].

While the above discussions focus on the quintet state, the reasoning is equally valid for other high-spin states, such as the septet.

\section{APPENDIX F: MAGNETOSPECTROSCOPY SIMULATIONS}

In Fig. 2(c), we presented the minimal energy spectrum of the DQD that explains the phase response of the resonator $\Delta \phi$ across an interdot charge transition as a function of the magnetic field intensity $B$ [see Fig. 2(a)]. In this appendix, we describe the Hamiltonian of the DQD that we used to obtain the eigenenergies and simulate the resonator response.

We consider independent spin manifolds, i.e., small spinorbit and hyperfine couplings between states with different total spin angular momentum $S$, and no coupling between states with the same $S$ but different magnetic number $m_{\mathrm{s}}$. In that scenario, the Hamiltonian becomes separable, and for the singlet manifold $(S=0)$, it reads

$$
H_{\mathrm{S}}=\left(\begin{array}{cc}
\frac{\varepsilon}{2} & \frac{\Delta_{\mathrm{S}}}{2} \\
\frac{\Delta_{\mathrm{S}}}{2} & -\frac{\varepsilon}{2}
\end{array}\right)
$$

where $\varepsilon$ is the energy detuning between dots and $\Delta_{\mathrm{S}}$ is the tunnel coupling between singlet states. For the triplet manifold $(S=1)$, we have

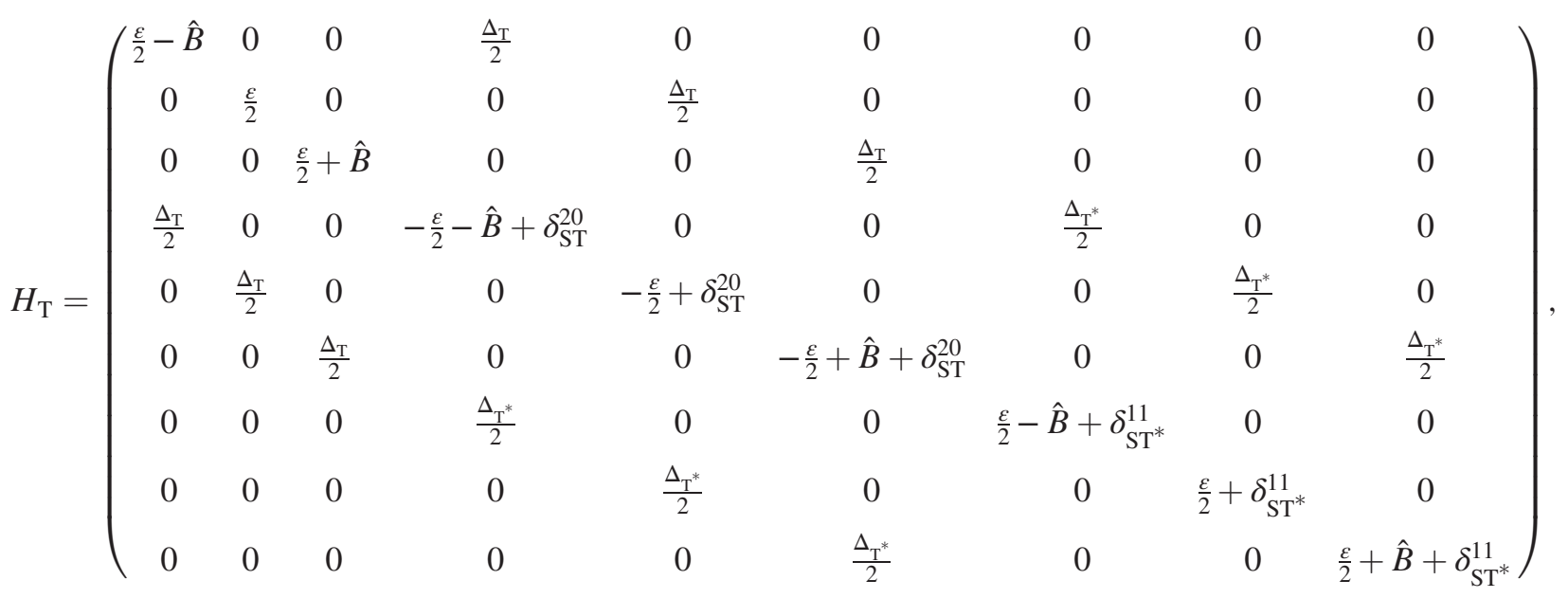


where $\hat{B}=g \mu_{\mathrm{B}} B, g$ is the electron $g$ factor, $\mu_{\mathrm{B}}$ is the Bohr magneton, $\Delta_{\mathrm{T}}\left[\Delta_{\mathrm{T}^{*}}\right]$ is the tunnel coupling between the $\mathrm{T}(2,14)$ and $\mathrm{T}(1,15)\left[\mathrm{T}^{*}(1,15)\right]$ triplet states, and $\delta_{\mathrm{ST}}^{20}\left[\delta_{\mathrm{ST}^{*}}^{11}\right]$ is the energy splitting between $\mathrm{S}(2,14)[\mathrm{S}(2,15)]$ and $\mathrm{T}(2,14)\left[\mathrm{T}^{*}(1,15)\right]$ at large positive [negative] detuning and $B=0$. Next, we have the quintet manifold with $S=2$ :

$H_{\mathrm{Q}}=\left(\begin{array}{cccccccccc}\frac{\varepsilon}{2}-2 \hat{B}+\delta_{\mathrm{SQ}}^{11} & 0 & 0 & 0 & 0 & \frac{\Delta_{\mathrm{Q}}}{2} & 0 & 0 & 0 & 0 \\ 0 & \frac{\varepsilon}{2}-\hat{B}+\delta_{\mathrm{SQ}}^{11} & 0 & 0 & 0 & 0 & \frac{\Delta_{\mathrm{Q}}}{2} & 0 & 0 & 0 \\ 0 & 0 & \frac{\varepsilon}{2}+\delta_{\mathrm{SQ}}^{11} & 0 & 0 & 0 & 0 & \frac{\Delta_{\mathrm{Q}}}{2} & 0 & 0 \\ 0 & 0 & 0 & \frac{\varepsilon}{2}+\hat{B}+\delta_{\mathrm{SQ}}^{11} & 0 & 0 & 0 & 0 & \frac{\Delta_{\mathrm{Q}}}{2} & 0 \\ 0 & 0 & 0 & 0 & \frac{\varepsilon}{2}+2 \hat{B}+\delta_{\mathrm{SQ}}^{11} & 0 & 0 & 0 & 0 & \frac{\Delta_{\mathrm{Q}}}{2} \\ \frac{\Delta_{\mathrm{Q}}}{2} & 0 & 0 & 0 & 0 & -\frac{\varepsilon}{2}-2 \hat{B}+\delta_{\mathrm{SQ}}^{20} & 0 & 0 & 0 & 0 \\ 0 & \frac{\Delta_{\mathrm{Q}}}{2} & 0 & 0 & 0 & 0 & -\frac{\varepsilon}{2}-\hat{B}+\delta_{\mathrm{SQ}}^{20} & 0 & 0 & 0 \\ 0 & 0 & \frac{\Delta_{\mathrm{Q}}}{2} & 0 & 0 & 0 & 0 & -\frac{\varepsilon}{2}+\delta_{\mathrm{SQ}}^{20} & 0 & 0 \\ 0 & 0 & 0 & \frac{\Delta_{\mathrm{Q}}}{2} & 0 & 0 & 0 & 0 & -\frac{\varepsilon}{2}+\hat{B}+\delta_{\mathrm{SQ}}^{20} & 0 \\ 0 & 0 & 0 & 0 & \frac{\Delta_{\mathrm{Q}}}{2} & 0 & 0 & 0 & 0 & -\frac{\varepsilon}{2}+2 \hat{B}+\delta_{\mathrm{SQ}}^{20}\end{array}\right)$,

where $\Delta_{\mathrm{Q}}$ is the quintet tunnel coupling and $\delta_{\mathrm{SQ}}^{11}\left[\delta_{\mathrm{SQ}}^{20}\right]$ is the energy splitting between $\mathrm{S}(1,15)[\mathrm{S}(2,14)]$ and $\mathrm{Q}(1,15)[\mathrm{Q}$ $(2,14)]$ at large negative [positive] detuning and $B=0$. Finally, the septet manifold $(S=3)$ can be described by

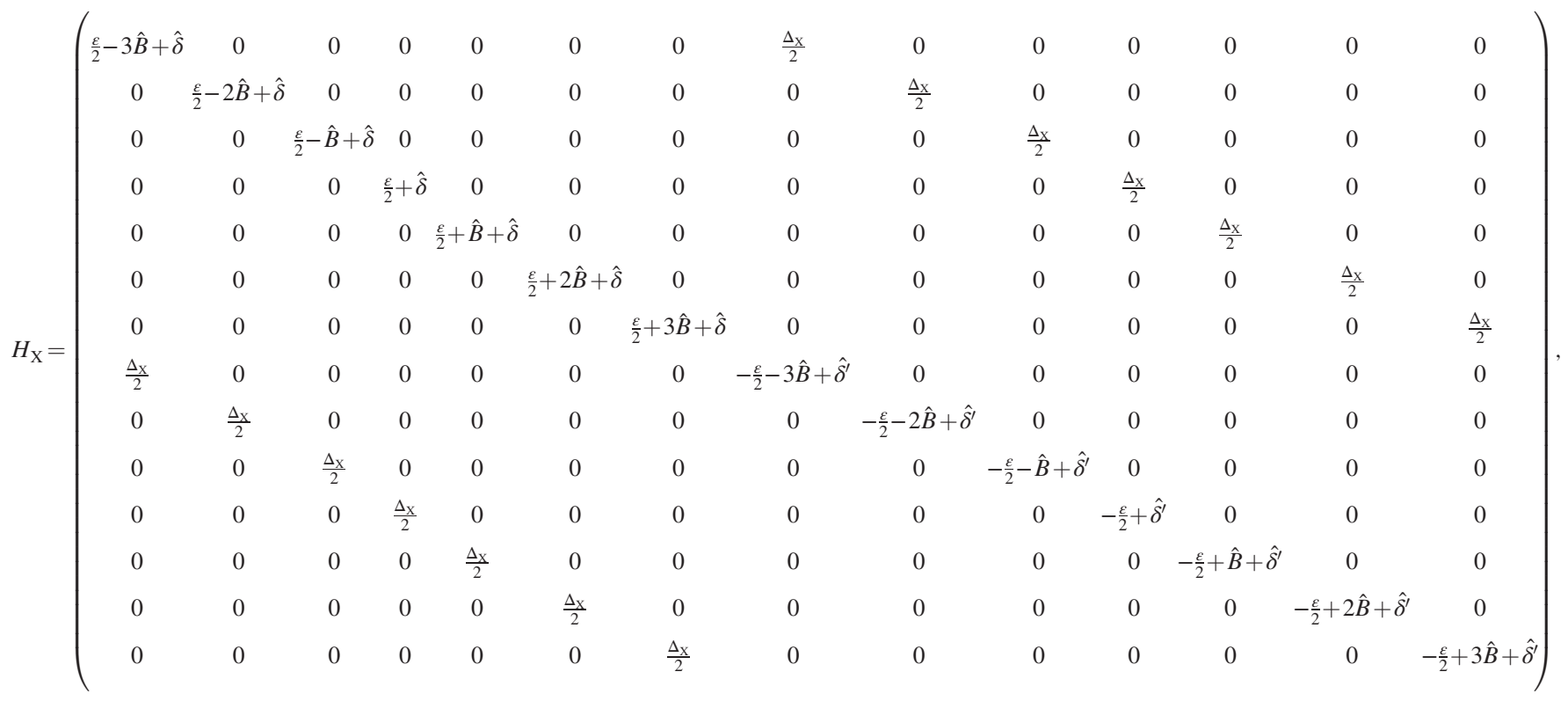

where $\Delta_{\mathrm{X}}$ is the septet tunnel coupling, $\hat{\delta}=\delta_{\mathrm{SX}}^{11}\left[\hat{\delta}^{\prime}=\delta_{\mathrm{SX}}^{20}\right]$ is the energy splitting between the $\mathrm{S}(1,15)[\mathrm{S}(2,14)]$ and $\mathrm{X}(1,15)$ $[\mathrm{X}(2,14)]$ at large negative [positive] detuning, and $B=0$. In order to simulate the dispersive response of this system as a function of $B$ [Fig. 2(b)], we extract the total quantum capacitance from [36]

$$
C_{\mathrm{Q}}=-\sum_{i}(e \alpha)^{2} \frac{\partial^{2} E_{i}}{\partial \varepsilon^{2}} P_{i}^{\mathrm{th}}
$$

where $E_{i}$ are the eigenenergies of the above Hamiltonians and $P_{i}^{\text {th }}$ is the thermal probability of the state $i$, 


$$
P_{i}^{\mathrm{th}}=\exp \left(-E_{i} / k_{\mathrm{B}} T\right) / Z
$$

Here, $k_{\mathrm{B}}$ is the Boltzmann constant, $T$ is the DQD temperature, and $Z$ is the partition function over all states.

[1] M. J. Y. Tayebjee, S. N. Sanders, E. Kumarasamy, L. M. Campos, M. Y. Sfeir, and D. R. McCamey, Quintet Multiexciton Dynamics in Singlet Fission, Nat. Phys. 13, 182 (2017).

[2] M. B. Smith and J. Michl, Singlet Fission, Chem. Rev. 110, 6891 (2010).

[3] H. Kim, K. Wang, Y. Nakajima, R. Hu, S. Ziemak, P. Syers, L. Wang, H. Hodovanets, J. D. Denlinger, P. M. R. Brydon et al., Beyond Triplet: Unconventional Superconductivity in a Spin-3/2 Topological Semimetal, Sci. Adv. 4, eaao4513 (2018).

[4] J. Yu and C.-X. Liu, Singlet-Quintet Mixing in Spin-Orbit Coupled Superconductors with $j=3 / 2$ Fermions, Phys. Rev. B 98, 104514 (2018).

[5] M. Swart, A. R. Groenhof, A. W. Ehlers, and K. Lammertsma, Validation of Exchange-Correlation Functionals for Spin States of Iron Complexes, J. Phys. Chem. A 108, 5479 (2004).

[6] S. V. Chapyshev, R. Walton, J. A. Sanborn, and P. M. Lahti, Quintet and Septet State Systems Based on Pyridylnitrenes: Effects of Substitution on Open-Shell High-Spin States, J. Am. Chem. Soc. 122, 1580 (2000).

[7] Y. Teki, S. Miyamoto, M. Nakatsuji, and Y. Miura, $\pi$-Topology and Spin Alignment Utilizing the Excited Molecular Field: Observation of the Excited High-Spin Quartet $(S=3 / 2)$ and Quintet $(S=2)$ States on Purely Organic $\pi$-Conjugated Spin Systems, J. Am. Chem. Soc. 123, 294 (2001).

[8] F. K. Malinowski, F. Martins, T. B. Smith, S. D. Bartlett, A. C. Doherty, P. D. Nissen, S. Fallahi, G. C. Gardner, M. J. Manfra, C. M. Marcus et al., Spin of a Multielectron Quantum Dot and Its Interaction with a Neighboring Electron, Phys. Rev. X 8, 011045 (2018).

[9] B.-B. Chen, B.-C. Wang, G. Cao, H.-O. Li, M. Xiao, G.-C. Guo, H.-W. Jiang, X. Hu, and G.-P. Guo, Spin Blockade and Coherent Dynamics of High-Spin States in a Three-Electron Double Quantum Dot, Phys. Rev. B 95, 035408 (2017).

[10] J. van der Heijden, T. Kobayashi, M. G. House, J. Salfi, S. Barraud, R. Laviéville, M. Y. Simmons, and S. Rogge, Readout and Control of the Spin-Orbit States of Two Coupled Acceptor Atoms in a Silicon Transistor, Sci. Adv. 4, eaat9199 (2018).

[11] R. C. C. Leon, C. H. Yang, J. C. C. Hwang, J. C. Lemyre, T. Tanttu, W. Huang, K. W. Chan, K. Y. Tan, F. E. Hudson, K. M. Itoh et al., Coherent Spin Control of $s$-, $p$-, $d$ - and f-Electrons in a Silicon Quantum Dot, Nat. Commun. 11, 797 (2020).

[12] A. M. Tyryshkin, S. Tojo, J. J. L. Morton, H. Riemann, N. V. Abrosimov, P. Becker, H.-J. Pohl, T. Schenkel, M. L. W. Thewalt, K. M. Itoh et al., Electron Spin Coherence Exceeding Seconds in High-Purity Silicon, Nat. Mater. 11, 143 (2012).
[13] K. Saeedi, S. Simmons, J. Z. Salvail, P. Dluhy, H. Riemann, N. V. Abrosimov, P. Becker, H.-J. Pohl, J. J. L. Morton, and M. L. W. Thewalt, Room-Temperature Quantum Bit Storage Exceeding 39 Minutes Using Ionized Donors in Silicon-28, Science 342, 830 (2013).

[14] R. Maurand, X. Jehl, D. Kotekar-Patil, A. Corna, H. Bohuslavskyi, R. Laviéville, L. Hutin, S. Barraud, M. Vinet, M. Sanquer et al., A CMOS Silicon Spin Qubit, Nat. Commun. 7, 13575 (2016).

[15] M. Veldhorst, H. G. J. Eenink, C. H. Yang, and A. S. Dzurak, Silicon CMOS Architecture for a Spin-Based Quantum Computer, Nat. Commun. 8, 1766 (2017).

[16] A. Crippa, R. Ezzouch, A. Aprá, A. Amisse, L. Houtin, B. Bertrand, M. Vinet, M. Urdampilleta, T. Meunier, M. Sanquer et al., Gate-Reflectometry Dispersive Readout and Coherent Control of a Spin Qubit in Silicon, Nat. Commun. 10, 2776 (2019).

[17] J. Yoneda, K. Takeda, T. Otsuka, T. Nakajima, M. R. Delbecq, G. Allison, T. Honda, T. Kodera, S. Oda, Y. Hoshi et al., A Quantum-Dot Spin Qubit with Coherence Limited by Charge Noise and Fidelity Higher than 99.9\%, Nat. Nanotechnol. 13, 102 (2018).

[18] W. Huang, C. H. Yang, K. W. Chan, T. Tanttu, B. Hensen, R. C. C. Leon, M. A. Fogarty, J. C. C. Hwang, F. E. Hudson, K. M. Itoh et al., Fidelity Benchmarks for Two-Qubit Gates in Silicon, Nature (London) 569, 532 (2019).

[19] T. F. Watson, S. G. Philips, E. Kawakami, D. R. Ward, P. Scarlino, M. Veldhorst, D. E. Savage, M. G. Lagally, M. Friesen, S. N. Coppersmith, M. A. Eriksson, and L. M. Vandersypen, A Programmable Two-Qubit Quantum Processor in Silicon, Nature (London) 555, 633 (2018).

[20] A. Morello, J. J. Pla, F. A. Zwanenburg, K. W. Chan, K. Y. Tan, H. Huebl, M. Möttönen, C. D. Nugroho, C. Yang, J. A. Van Donkelaar, A. D. Alves, D. N. Jamieson, C. C. Escott, L. C. Hollenberg, R. G. Clark, and A. S. Dzurak, SingleShot Readout of an Electron Spin in Silicon, Nature (London) 467, 687 (2010).

[21] M. D. Schroer, M. Jung, K. D. Petersson, and J. R. Petta, Radio Frequency Charge Parity Meter, Phys. Rev. Lett. 109, 166804 (2012).

[22] C. H. Yang, R. C. C. Leon, J. C. C. Hwang, A. Saraiva, T. Tanttu, W. Huang, J. C. Lemyre, K. W. Chan, K. Y. Tan, F. E. Hudson et al., Silicon Quantum Processor Unit Cell Operation above One Kelvin, Nature (London) 580, 350 (2020).

[23] R. Zhao, T. Tanttu, K. Y. Tan, B. Hensen, K. W. Chan, J. C. C. Hwang, R. C. C. Leon, C. H. Yang, W. Gilbert, F. E. Hudson et al., Single-Spin Qubits in Isotopically Enriched Silicon at Low Magnetic Field, Nat. Commun. 10, 5500 (2019).

[24] C. Kloeffel and D. Loss, Prospects for Spin-Based Quantum Computing in Quantum Dots, Annu. Rev. Condens. Matter Phys. 4, 51 (2013).

[25] M. A. Fogarty, K. W. Chan, B. Hensen, W. Huang, T. Tanttu, C. H. Yang, A. Laucht, M. Veldhorst, F. E. Hudson, K. M. Itoh et al., Integrated Silicon Qubit Platform with SingleSpin Addressability, Exchange Control and Single-Shot Singlet-Triplet Readout, Nat. Commun. 9, 4370 (2018).

[26] P. Harvey-Collard, B. D’Anjou, M. Rudolph, N. T. Jacobson, J. Dominguez, G. A. Ten Eyck, J. R. Wendt, T. Pluym, 
M. P. Lilly, W. A. Coish et al., High-Fidelity Single-Shot Readout for a Spin Qubit via an Enhanced Latching Mechanism, Phys. Rev. X 8, 021046 (2018).

[27] A. C. Betz, R. Wacquez, M. Vinet, X. Jehl, A. L. Saraiva, M. Sanquer, A. J. Ferguson, and M. F. Gonzalez-Zalba, Dispersively Detected Pauli Spin-Blockade in a Silicon Nanowire Field-Effect Transistor, Nano Lett. 15, 4622 (2015).

[28] D. Culcer, A. L. Saraiva, B. Koiller, X. Hu, and S. Das Sarma, Valley-Based Noise-Resistant Quantum Computation Using Si Quantum Dots, Phys. Rev. Lett. 108, 126804 (2012).

[29] A. P. Higginbotham, F. Kuemmeth, M. P. Hanson, A. C. Gossard, and C. M. Marcus, Coherent Operations and Screening in Multielectron Spin Qubits, Phys. Rev. Lett. 112, 026801 (2014).

[30] E. Nielsen, E. Barnes, J. P. Kestner, and S. D. Sarma, SixElectron Semiconductor Double Quantum Dot Qubits, Phys. Rev. B 88, 195131 (2013).

[31] D. Weinmann, W. Häusler, and B. Kramer, Spin Blockades in Linear and Nonlinear Transport through Quantum Dots, Phys. Rev. Lett. 74, 984 (1995).

[32] K. D. Petersson, C. G. Smith, D. Anderson, P. Atkinson, G. A. C. Jones, and D. A. Ritchie, Charge and Spin State Readout of a Double Quantum Dot Coupled to a Resonator, Nano Lett. 10, 2789 (2010).

[33] J. I. Colless, A. C. Mahoney, J. M. Hornibrook, A. C. Doherty, H. Lu, A. C. Gossard, and D. J. Reilly, Dispersive Readout of a Few-Electron Double Quantum Dot with Fast rf Gate Sensors, Phys. Rev. Lett. 110, 046805 (2013).

[34] I. Ahmed, J. A. Haigh, S. Schaal, S. Barraud, Y. Zhu, C. M. Lee, M. Amado, J. W. A. Robinson, A. Rossi, J. J. L. Morton, and M.F. Gonzalez-Zalba, Radio-Frequency Capacitive Gate-Based Sensing, Phys. Rev. Applied 10, 014018 (2018).

[35] B. Voisin, R. Maurand, S. Barraud, M. Vinet, X. Jehl, M. Sanquer, J. Renard, and S. De Franceschi, Electrical Control of g-Factor in a Few-Hole Silicon Nanowire MOSFET, Nano Lett. 16, 88 (2015).

[36] R. Mizuta, R. M. Otxoa, A. C. Betz, and M. F. GonzalezZalba, Quantum and Tunneling Capacitance in Charge and Spin Qubits, Phys. Rev. B 95, 045414 (2017).

[37] The charge stability diagram in Fig. 1(b) is stitched together by 49 individual diagrams, each of dimension $0.1 \mathrm{~V} \times 0.1 \mathrm{~V}$.

[38] W. V. D. Wiel, S. D. Franceschi, J. Elzerman, T. Fujisawa, S. Tarucha, and L. Kouwenhoven, Electron Transport through Double Quantum Dots, Rev. Mod. Phys. 75, 1 (2002).

[39] N. S. Lai, W. H. Lim, C. H. Yang, F. A. Zwanenburg, W. A. Coish, F. Qassemi, A. Morello, and A. S. Dzurak, Pauli Spin Blockade in a Highly Tunable Silicon Double Quantum Dot, Sci. Rep. 1, 110 (2011).

[40] J. R. Petta, A. C. Johnson, J. M. Taylor, E. A. Laird, A. Yacoby, M. D. Lukin, C. M. Marcus, M. P. Hanson, and A. C. Gossard, Coherent Manipulation of Coupled Electron Spins in Semiconductor Quantum Dots, Science 309, 2180 (2005).

[41] A. West, B. Hensen, A. Jouan, T. Tanttu, C.-H. Yang, A. Rossi, M. F. Gonzalez-Zalba, F. Hudson, A. Morello, D. J.
Reilly et al., Gate-Based Single-Shot Readout of Spins in Silicon, Nat. Nanotechnol. 14, 437 (2019).

[42] M. G. House, T. Kobayashi, B. Weber, S. J. Hile, T. F. Watson, J. Van Der Heijden, S. Rogge, and M. Y. Simmons, Radio Frequency Measurements of Tunnel Couplings and Singlet-Triplet Spin States in Si:P Quantum Dots, Nat. Commun. 6, 8848 (2015).

[43] A. J. Landig, J. V. Koski, P. Scarlino, C. Reichl, W. Wegscheider, A. Wallraff, K. Ensslin, and T. Ihn, Microwave-Cavity-Detected Spin Blockade in a FewElectron Double Quantum Dot, Phys. Rev. Lett. 122, 213601 (2019).

[44] See Supplemental Material at http://link.aps.org/ supplemental/10.1103/PhysRevX.10.041010 for magnetospectroscopy showing evidence of quintet spin states in a different, similar device.

[45] D. J. Ibberson, L. Bourdet, J. C. Abadillo-Uriel, I. Ahmed, S. Barraud, M. J. Calderón, Y. M. Niquet, and M. F. Gonzalez-Zalba, Electric-Field Tuning of the Valley Splitting in Silicon Corner Dots, Appl. Phys. Lett. 113, 053104 (2018).

[46] A. Corna, L. Bourdet, R. Maurand, A. Crippa, D. KotekarPatil, H. Bohuslavskyi, R. Laviéville, L. Hutin, S. Barraud, $\mathrm{X}$. Jehl et al., Electrically Driven Electron Spin Resonance Mediated by Spin-Valley-Orbit Coupling in a Silicon Quantum Dot, npj Quantum Inf. 4, 6 (2018).

[47] L. Bourdet and Y.-M. Niquet, All-Electrical Manipulation of Silicon Spin Qubits with Tunable Spin-Valley Mixing, Phys. Rev. B 97, 155433 (2018).

[48] M. Urdampilleta, D. J. Niegemann, E. Chanrion, B. Jadot, C. Spence, P.-A. Mortemousque, C. Bäuerle, L. Hutin, B. Bertrand, S. Barraud et al., Gate-Based High Fidelity Spin Readout in a CMOS Device, Nat. Nanotechnol. 14, 737 (2019).

[49] L. Hutin et al., Gate Reflectometry for Probing Charge and Spin States in Linear Si MOS Split-Gate Arrays, in 2019 IEEE International Electron Devices Meeting (IEDM) (2019), pp. 37.7.1-37.7.4, https://doi.org/10.1109/ IEDM19573.2019.8993580.

[50] Z. Shi, C. B. Simmons, J. R. Prance, J. K. Gamble, T. S. Koh, Y.-P. Shim, X. Hu, D. E. Savage, M. G. Lagally, M. A. Eriksson et al., Fast Hybrid Silicon Double-Quantum-Dot Qubit, Phys. Rev. Lett. 108, 140503 (2012).

[51] M. F. Gonzalez-Zalba, S. Barraud, A. J. Ferguson, and A. C. Betz, Probing the Limits of Gate-Based Charge Sensing, Nat. Commun. 6, 6084 (2015).

[52] P. Pakkiam, A. V. Timofeev, M. G. House, M. R. Hogg, T. Kobayashi, M. Koch, S. Rogge, and M. Y. Simmons, SingleShot Single-Gate rf Spin Readout in Silicon, Phys. Rev. X 8 , 041032 (2018).

[53] J. R. Prance, Z. Shi, C. B. Simmons, D. E. Savage, M. G. Lagally, L. R. Schreiber, L. M. K. Vandersypen, M. Friesen, R. Joynt, S. N. Coppersmith et al., Single-Shot Measurement of Triplet-Singlet Relaxation in a Si/SiGe Double Quantum Dot, Phys. Rev. Lett. 108, 046808 (2012).

[54] http://mos-quito.eu. 